\title{
E-Commerce Precision Marketing Model Based on Convolutional Neural Network
}

\author{
Xia Liu ii \\ Shandong University of Finance and Economics, Shandong, Jinan, China \\ Correspondence should be addressed to Xia Liu; 20088532@sdufe.edu.cn
}

Received 23 December 2021; Revised 18 January 2022; Accepted 26 January 2022; Published 7 March 2022

Academic Editor: Hangjun Che

Copyright () 2022 Xia Liu. This is an open access article distributed under the Creative Commons Attribution License, which permits unrestricted use, distribution, and reproduction in any medium, provided the original work is properly cited.

\begin{abstract}
With the rapid development of network and informatization of the consumer market in my country, the application and maturity of technologies such as the Internet, terminal equipment, logistics, and payment and the continuous improvement of people's consumption concepts, online shopping has gradually become the mainstream purchase method for Chinese consumers, and e-commerce has gradually become one of the important driving forces to promote the sustained and vigorous development of China's economy. Under the traditional marketing model, companies do not fully understand the needs of users. The sales staff's thinking is only how to sell products to users. They do not know the specific consumer needs, so they can only focus on the product. Based on these foundations, this research uses convolutional neural networks and applies this model to precision marketing to obtain accurate portraits of consumers, thereby increasing the company's turnover. After comparing different models and conducting some experiments, it is concluded that (1) through the collection and analysis of W enterprise data, the training and testing conditions of the CNN model, LSTM model, LSTM attention model, and CNN + LSTM attention model are compared. It is concluded that the CNN + LSTM attention model and the LSTM attention model perform better, and the accuracy of testing and training is higher. (2) Through the fitting of the model, it is found that $\operatorname{Sn}(\%)=70.71, \operatorname{Sp}(\%)=86.25, \operatorname{Acc}(\%)=81.07$, and MCC $=0.752$ of the CNN + LSTM attention model are the best fitting models. The men and women stratification and gender stratification of users are predicted, and it is found that men in the $\mathrm{W}$ company are the main purchasing power, and in the age stratification, it is found that the population of 41-50 accounts for the highest proportion. (3) The average accuracy rate of the LSTM attention model is as high as $66.6 \%$, the average recall rate is $82.3 \%$, and the F1 score is $73.1 \%$. This model has met expectations for precision marketing forecasts. (4) Using the CNN + LSTM attention model to predict the marketing input for the next year, it is found that the use of precision marketing will increase the profit of W company. The average annual data show that the monthly revenue of precision marketing has increased by $73.5 \%$.
\end{abstract}

\section{Introduction}

In the past ten years, with the rapid development of network and informatization of China's consumer market, the application and maturity of technologies such as the Internet, terminal equipment, logistics, and payment and the continuous improvement of people's consumption concepts, online shopping has gradually become the mainstream purchase method for Chinese consumers. E-commerce has gradually become one of the important driving forces to promote the sustained and vigorous development of China's economy $[1,2]$. Based on the analysis and statistics of the marketing models that currently exist in our society, it is concluded that the growth rate of e-commerce online marketing is getting faster and faster. The refined and precise marketing model will be the mainstream model in the future. Literature [3] only makes good use of big data to paint portraits of consumers. The precision marketing model can greatly promote offline marketing and online marketing. The function of marketing is to act as a communication bridge between the company and consumers. It can not only help consumers understand the company and make these consumers become loyal customers of the company but also play a role in publicity, allowing the company to better understand its audience. Therefore, it is necessary to learn and improve marketing concepts and transform from ordinary 
marketing to precision marketing. Precision marketing is a concept that may help promote collective thinking and understanding of the criteria used for segmentation and positioning. The purpose is to better serve customers, thereby highlighting the competitiveness of products and bringing profits to the company. Only by formulating sales strategies from person to person can the effect of precision marketing be maximized. Control the results and costs of communication as much as possible, give scientific standards, and avoid randomness. Convolutional neural networks are used to predict the detection index system of multilabel systems. The prediction model is superior to other existing prediction models in almost all five indicators of performance. The most outstanding performance in the "absolute truth" rate and "absolute truth" rate [4]. A multilabel classifier system is based on deep learning features of convolutional neural network to infer the classification of goods. The system is based on the two-dimensional representation of the sample: first, obtain a one-dimensional feature vector, extract the characteristics of the marketing amount and marketing model, find out the interaction and the structure and feature similarity information with other products of different categories, and then reshape the original one-dimensional feature vector to obtain a twodimensional matrix table of commodities. Finally, using feature extractors, two general classifiers designed for multilabel classification are trained using deep learning features. The scores obtained are fused by the average rule $[5,6]$. With the continuous breakthroughs in computer technology, deep neural networks and other technologies are becoming more and more widely used in our daily life applications. People process the acquired information with computer algorithms. Convolutional neural networks (CNN) are currently the mainstream computer network analysis means. Convolutional neural networks can be used to automatically learn structured data, extract effective features, and then use the extracted effective features to make predictions and make correct decisions, which can help manage company employees, predict consumer preferences, etc. Predictive classification of unknown consumers is of great significance to existing research. The ATC system is a multilabel classification system that classifies consumers according to their consumption preferences. The system includes five levels, and each level includes several levels; the first level includes 14 main overlapping categories. The ATC classification system also considers the distribution of population characteristics, brings into the model effect characteristics, and predicts unknown problems in its category. Such predictions can be used not only to infer the active ingredients of system performance but also to infer other possible active ingredients. Due to the high variability of samples and the overlap between classes, the problem of automatic prediction is very random, and there may be multiple prediction deviations and the complexity of machine learning. Convolutional neural networks are also widely used in the field of biomedical engineering. Medical image analysis is one of the most popular research and development fields. Deep learning has been successfully used as a machine learning tool. Neural networks have the ability to automatically learn features [7]. The rapid growth of data has enabled statistical modeling and machine learning methods to predict the information of some compounds in bioinformatics and chemoinformatics and contribute to various applications of metabolic engineering and drug discovery [8]. Convolutional neural networks have been successfully applied to network big data predictions. Using four different experimental network models and dual graphs with different sparsity and degree distributions, high prediction performance is achieved in the case of relatively dense, but the performance becomes worse in the case of extreme sparseness. Human decision-making processes usually rely on the use of visual information from different perspectives or perspectives. However, in image classification based on machine learning, we usually only infer the category of the object from a single image showing the object. Especially for challenging classification problems, the visual information conveyed by a single image may not be enough to make accurate decisions. The optimization scheme relies on the fusion of visual information captured by images depicting the same object from multiple angles [9]. Convolutional neural networks are used to extract and encode visual features from multiple views, to fuse this information to study the feature maps of different network depths for fusion convolution, to fuse potential bottlenecks before classification, and to score fusion. These strategies were systematically evaluated on three data sets from different fields. The discovery emphasizes the benefits of integrating information fusion into the network instead of performing it through the postprocessing of classification scores [10-13]. A case study that has been trained proves that the network can be easily expanded through the best fusion strategy, which is much better than other methods. The CNN model can be used to decode the hidden focus of attention related to EEG events in the object selection process. It compares the performance of $\mathrm{CNN}$ and the commonly used linear discriminant analysis (LDA) classifier, applies it to different dimensional data sets, and analyzes the transfer learning ability. Using CNN can conduct in-depth analysis of e-commerce data, convert the characteristics of each product into recognizable computer instructions, and use these characteristics to predict sales. The impact of individual model components can be verified by systematically changing the model, and the saliency map can be used as a tool to visualize the spatial and temporal characteristics that drive the output of the model. The effect of different attribute training sets on the sparse rate of the CNN output feature matrix is verified, and the improved Grad-CAM algorithm is used to train the key features to improve the stability and accuracy of the CNN model. Convolutional neural networks were originally discovered in biological laboratories. They were computer simulations and transformations of neural networks of humans or animals with brains. With the continuous exploration of science and technology by human beings, CNN is also undergoing continuous innovation and transformation, with more extensive applications and continuous enhancement of computing capabilities [14, 15]. 


\section{Materials and Methods}

2.1. Convolutional Neural Network (CNN). The basic composition of the existing neural network model group is composed of the following parts: the input layer $(100 \times 1)$ is used for the input of the original data grid conversion and the convolutional layer 1 is the basic data grid layer of the model. Data filtering and sorting play an important role, and the specific parameters are shown in Figure 1. Convolutional layer 2 (Conv2) is to perform secondary sorting and reverse confirmation on the data grid of the first layer [16-18]. The residual network and the expansion convolution are performed in the stretching layer, and the accuracy of the ConvNet architecture predicting contact is judged by the F1 connection layer. The basic flow of the experiment is shown in Figure 1.

2.2. Data Grid Conversion. Data grid conversion is to classify data in ascending dimensions to facilitate data extraction for subsequent research. Use the DNCON2 data set (V1, V2, and V3) for training and test on the CASP12 data set (V4, $\mathrm{V} 5$, and V6). The structural equation model is shown in Figure 2, which has strong connections and close connections. 'S association. A, B, and $\mathrm{C}$ are hidden variables, and $\mathrm{A}$ and $\mathrm{B}$ correspond to neurons in the convolutional layer. $\mathrm{Y} 1$ and Y2 are also observed variables. Figure 3 shows a convolution process diagram with a convolution kernel size of $1 \times 3$. This convolution process can simulate the convolution process of the first two layers, and the input related variables can be constructed as latent factors.

2.3. Precision Marketing and Convolutional Neural Network. In the $\mathrm{W}$ enterprise, a large amount of personal data is collected, extracted from the enterprise database, and then simulated by a convolutional neural network to simulate accurate consumer portraits. For a small number of anonymous users who cannot obtain accurate data, through simulation and prediction of previous data sets, the hidden data are extracted by looping and replying experiments. Integrate centralized data resources, establish an enterpriselevel big data center, and realize "normalization" and "resourceization." Convolutional neural networks can be used to assist companies in precision marketing models. The general process is to sort out the collected raw data and check the data, including the adjustment of the content format and the change of logic errors. In the rewriting of the basic label, the basic customer information is rewritten, and the threshold is set [19-21]. Perform label classification on the basis of basic label rewriting, based on characteristics such as crowd consumption habits. Then, use the convolutional neural network to predict the customer's behavior to form a three-dimensional label. The last is the output of the results, providing customers with personalized services and exporting detailed information items.

\section{Application of Convolutional Neural Network in Precision Marketing}

3.1. CNN Model

$$
\begin{aligned}
& \mu_{1}=v_{1} w_{1}, v_{2} w_{2}, v_{3} w_{3}, \\
& \mu_{2}=v_{4} w_{1}, v_{5} w_{2}, v_{6} w_{3} .
\end{aligned}
$$

Euclidean distance

$$
d_{i j}=\left(\sum_{k=1}^{n}\left(X_{k i}-X_{k i}\right)^{2}\right)^{1 / 2}
$$

Pearson correlation distance

$$
d_{i j}=1-\left|\rho_{1} A_{i}\right| \text {. }
$$

Let $D_{X}$ denote a matrix composed of elements $d_{i j}$ :

$$
p_{i j}=\sqrt{\left(r_{i}-r_{j}\right)^{2}+\left(c_{i}-c_{j}\right)^{2}} \text {. }
$$

Let $D_{Y}$ represent the matrix composed of elements $p_{i j}$ :

$$
\begin{aligned}
& \widetilde{D}_{x}=\frac{D_{x}}{\max \left(D_{x}\right)}, \\
& \widetilde{D}_{y}=\frac{D_{y}}{\max \left(D_{y}\right)} .
\end{aligned}
$$

Formula (5) calculates the number of users before correction, and formula (6) calculates the number of users after correction.

The convolution operation can be expressed by the following formula:

$$
Y^{[l]}=f\left(\sum_{n=1}^{n^{[l]}} W^{[l],}+b^{[l]}\right),
$$

where $y_{c}$ represents the marketing forecast profit, and the calculation method is as follows:

$$
y_{c}=\frac{\exp \left(z_{c}\right)}{\sum_{j} \exp \left(z_{j}\right)}, \quad c=1,2, \ldots, c
$$

\subsection{LSTM Model}

$$
\mathrm{Y}_{\text {conv }}=f\left(\sum_{j=0}^{J=1} \sum_{i=0}^{I=1} x_{m+i, n+j} w_{i j}+b\right)
$$

The value range of $n$ in formula (9) is $(0, n)$.

Among them, the interval of the activation parameter $m$ is between positive and negative $m, f$ is the activation function; $b$ is the additional offset (or offset); $\mathrm{Y}_{\text {conv }}$ is its output. 


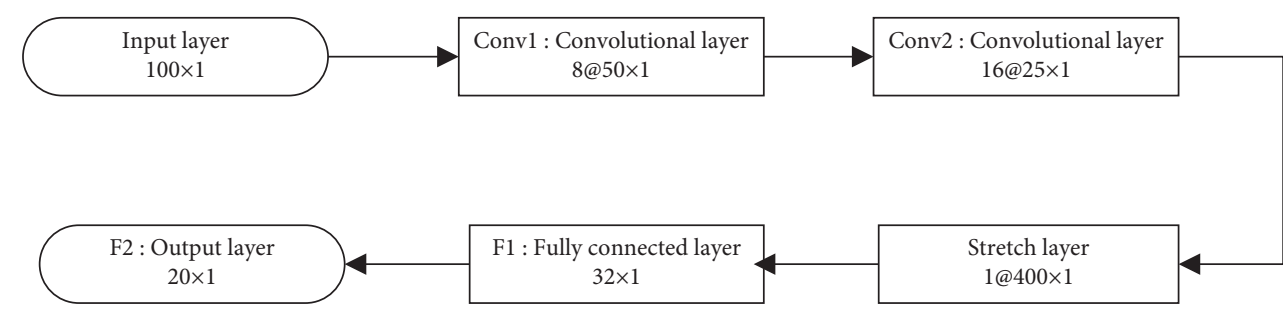

Figure 1: Convolutional neural network model.

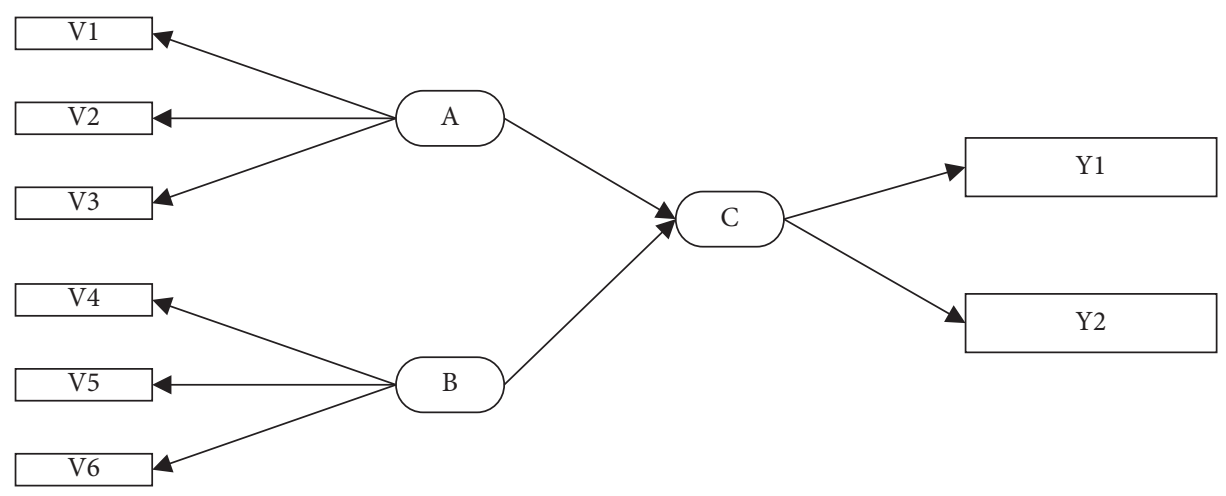

FIgURE 2: Structural equation simulation path diagram.

\begin{tabular}{|c|c|c|c|c|c|c|c|}
\hline V1 & V2 & V3 & \multicolumn{4}{|c|}{} & $\mu 1$ \\
\hline V4 & V5 & V6 & & W1 & W2 & W3 & \\
\hline
\end{tabular}

Figure 3: Convolution process with a $1 \times 3$ convolution kernel.

$$
F_{i}=\frac{1}{N}=\sum_{x=1}^{N} f_{i}(x)
$$

where $f_{i}(x)$ is the monthly profit income of $\mathrm{W}$ enterprise and $N$ is the quarterly total profit income.

$$
s_{c}=\sum_{i=1}^{M} F_{i} \times w_{i}^{c}
$$

The output of Softmax $\sigma$ (S prediction result represents the gender and age of the consumer group).

$$
\sigma\left(S_{c}\right)=\frac{e^{s_{c}}}{\sum_{j=1}^{c} e^{s_{j}}} \text { for } c=1, \ldots, c, s=\left(S_{1}, \ldots, S_{c}\right) \in \mathbb{R}^{c} .
$$

In order to avoid overfitting, a regularized model is used to constrain

$$
\text { loss }=-\frac{1}{n} \sum_{n=1}^{N}\left[y_{n} \log \hat{y}_{n}+\left(1-y_{n}\right) \log \left(1-y_{n}\right)\right]+\lambda\|w\|^{2} \text {. }
$$

Comprehensive judgment of the model

$$
\begin{aligned}
s_{c} & =\sum_{i=1}^{M} F_{i} \times w_{i}^{c}=\frac{1}{N} \sum_{x=1}^{N} \sum_{i=1}^{M} w_{i}^{c} \times f_{i}(x), \\
M_{c}(x) & =\sum_{i=1}^{M} w_{i}^{c} \times P_{i}(x),
\end{aligned}
$$

The specific formula for the maximum sales of an enterprise is as follows:

$$
f_{\text {pool }}=\operatorname{Max}\left(x_{m, n}, x_{m, n+l}, x_{m+l, n+l}\right),
$$

where $f_{\text {pool }}$ is the predicted result of the largest sales.

ReLu is a consumer characteristic factor:

$$
f_{(x)}=\max (0, x) .
$$

3.3. LSTM Attention Model. Correlation coefficient of two vectors: 


$$
\begin{aligned}
\operatorname{PCC}(\vec{X}, \vec{Y},) & =\frac{s_{X} \vec{Y}}{s_{\vec{X}} s_{\vec{Y}}}, \\
s_{\vec{X}} \vec{Y} & =\frac{1}{N-1} \sum_{k=1}^{N}\left(x_{k}-\bar{x}\right)\left(y_{k}-\bar{y}\right), \\
s_{\vec{X}} & =\sqrt{\frac{1}{N-1} \sum_{k=1}^{N}\left(x_{k}-\bar{x}\right)^{2},} \\
s_{\vec{Y}} & =\sqrt{\frac{1}{N-1} \sum_{k=1}^{N}\left(y_{k}-\bar{y}\right)^{2},} \\
\bar{x} & =\frac{1}{N} \sum_{k=1}^{N} x_{k}, \\
\bar{y} & =\frac{1}{N} \sum_{k=1}^{N} y_{k} .
\end{aligned}
$$

Here, $x_{k}$ and $y_{k}$ are the $k$ th element of the vector. Finally, the precise demand profile of consumers is simulated [22].

3.4. CNN + LSTM Attention Model. Euclidean distance for two features

$$
E D(\vec{X}, \vec{Y})=\sqrt{\sum_{k=1}^{N}\left(x_{k}-y_{k}\right)^{2}} .
$$

The maximum distance is recorded as

$$
\max M D_{i}=E D_{i}(1 \leq i \leq M) \text {. }
$$

The F-score of consumer demand characteristics is defined as

$$
F-\operatorname{score}(j)=\frac{\left(\bar{x}_{j}^{(+)}-\bar{x}_{j}\right)^{2}+\left(\bar{x}_{j}^{(-)}-\bar{x}_{j}\right)^{2}}{1 / m^{+}-1 \sum_{k=1}^{m^{+}}\left(\bar{x}_{k, j}^{(+)}-\bar{x}_{j}^{(+)}\right)^{2}+1 / m^{-}-1 \sum_{k=1}^{m^{-}}\left(\bar{x}_{k, j}^{(-)}-\bar{x}_{j}^{(-)}\right)^{2}}
$$

$\bar{x}_{j}, \bar{x}_{j}^{(+)}$, and $\bar{x}_{j}{ }^{(-)}$, respectively, represent the average value of all, positive, and negative predictions and actual eigenvalues. $m^{+}$and $m^{-}$are the error interval between forecast and actual. The larger the value, the more obvious the consumer's demand characteristics, and $q_{i}$ is the frequency of appearance.

$$
q_{i}=\frac{m_{i}}{M}
$$

$M$ is the total number of consumer features in all training data sets [23]. The probability in the male population and the female population can be defined as

$$
\begin{aligned}
p\left(n_{1, j}\right) & =\sum_{m=n_{1 j}}^{N_{j}} \frac{N_{j} !}{m !\left(N_{j}-m\right)} q_{i}^{m}\left(1-q_{i}\right)^{N_{j}-m}, \\
p\left(n_{-1, j}\right) & =\sum_{m=n_{-1 j}}^{N_{j}} \frac{N_{j} !}{m !\left(N_{j}-m\right)} q_{i}^{m}\left(1-q_{i}\right)^{N_{j}-m} .
\end{aligned}
$$

where $n_{1, j}$ and $n_{-1, j}$ is the number of users.

Finally, according to the following formula, the company's turnover through precision marketing is predicted:

$$
P_{j}=\min \left(p\left(n_{1, j}\right), p\left(n_{-1, j}\right)\right. \text {. }
$$

\section{Simulation Experiment}

4.1. Training and Testing of the Model. As shown in Table 1, the training and testing of the CNN model, LSTM model, LSTM attention model, and CNN + LSTM attention model are compared in detail. The data source is the commercial marketing data of W company. After filtering the data, we input it to our model. Choose from July to July, July to August, July to September, July to October, July to November, August to August, August to September, August to October, August to November, and September to September. Data from different months are used; data from September to October are used for testing, and data from September to November are used for training. Based on the results of several models, the CNN + LSTM attention model and the LSTM attention model perform better, and the accuracy of testing and training is higher.

Figure 4 visualizes the training and testing comparison of the CNN model. The training and testing comparison of the LSTM Train model is shown in Figure 5. Figure 6 is the training and testing comparison of the LSTM attention model after LSTM optimization. The comparison of training and testing of the CNN+ LSTM attention model is shown in Figure 7. The comprehensive icon result CNN + LSTM attention model is the optimal model [24-27]. 
TABLE 1: Comparison of training and testing of different models.

\begin{tabular}{|c|c|c|c|c|c|c|c|c|}
\hline \multirow[t]{2}{*}{ Month } & \multicolumn{2}{|c|}{$\mathrm{CNN}$} & \multicolumn{2}{|c|}{ LSTM } & \multicolumn{2}{|c|}{ LSTM attention } & \multicolumn{2}{|c|}{$\begin{array}{c}\mathrm{CNN}+\mathrm{LSTM} \\
\text { attention }\end{array}$} \\
\hline & Train & Test & Train & Test & Train & Test & Train & Test \\
\hline $7-7$ & 0.75 & 0.74 & 0.69 & 0.68 & 0.71 & 0.73 & 0.72 & 0.72 \\
\hline $7-8$ & 0.61 & 0.62 & 0.53 & 0.52 & 0.58 & 0.56 & 0.59 & 0.57 \\
\hline $7-9$ & 0.62 & 0.63 & 0.61 & 0.6 & 0.69 & 0.68 & 0.69 & 0.69 \\
\hline $7-10$ & 0.65 & 0.64 & 0.6 & 0.59 & 0.69 & 0.68 & 0.67 & 0.69 \\
\hline $7-11$ & 0.61 & 0.62 & 0.65 & 0.64 & 0.66 & 0.65 & 0.66 & 0.65 \\
\hline $8-8$ & 0.75 & 0.76 & 0.77 & 0.76 & 0.78 & 0.77 & 0.77 & 0.79 \\
\hline $8-9$ & 0.81 & 0.8 & 0.79 & 0.78 & 0.8 & 0.81 & 0.84 & 0.8 \\
\hline $8-10$ & 0.76 & 0.77 & 0.75 & 0.74 & 0.76 & 0.78 & 0.77 & 0.77 \\
\hline $8-11$ & 0.75 & 0.74 & 0.72 & 0.71 & 0.73 & 0.74 & 0.73 & 0.74 \\
\hline $9-9$ & 0.85 & 0.84 & 0.8 & 0.79 & 0.81 & 0.81 & 0.83 & 0.79 \\
\hline $9-10$ & 0.74 & 0.73 & 0.75 & 0.74 & 0.76 & 0.74 & 0.75 & 0.76 \\
\hline 9-11 & 0.65 & 0.69 & 0.68 & 0.67 & 0.69 & 0.7 & 0.68 & 0.7 \\
\hline
\end{tabular}

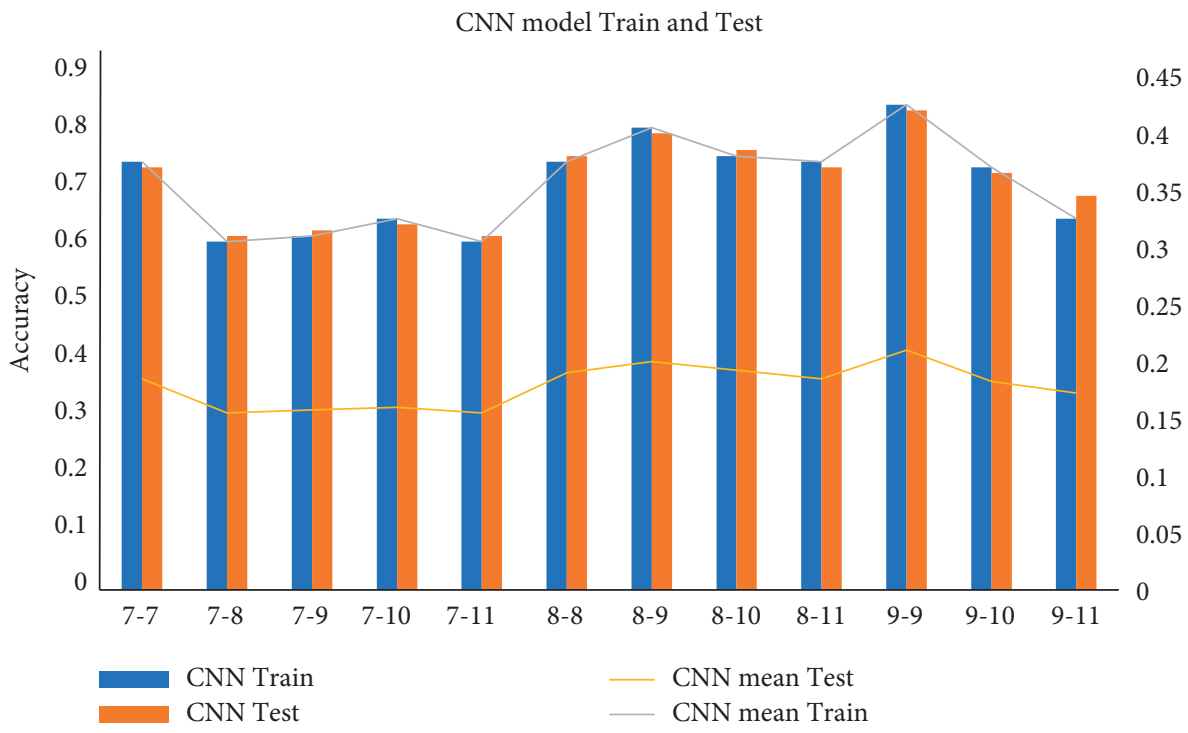

FIgURE 4: Comparison of training and testing of CNN model.

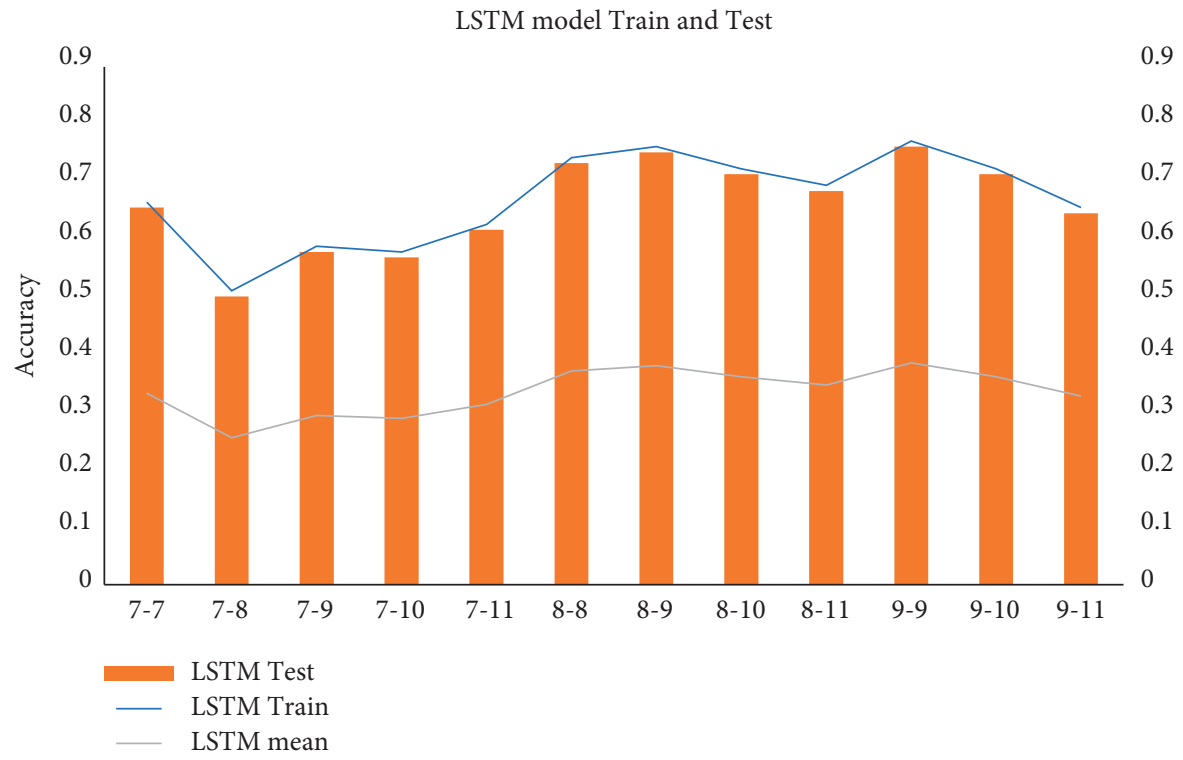

FIgure 5: Comparison of training and testing of LSTM train model. 


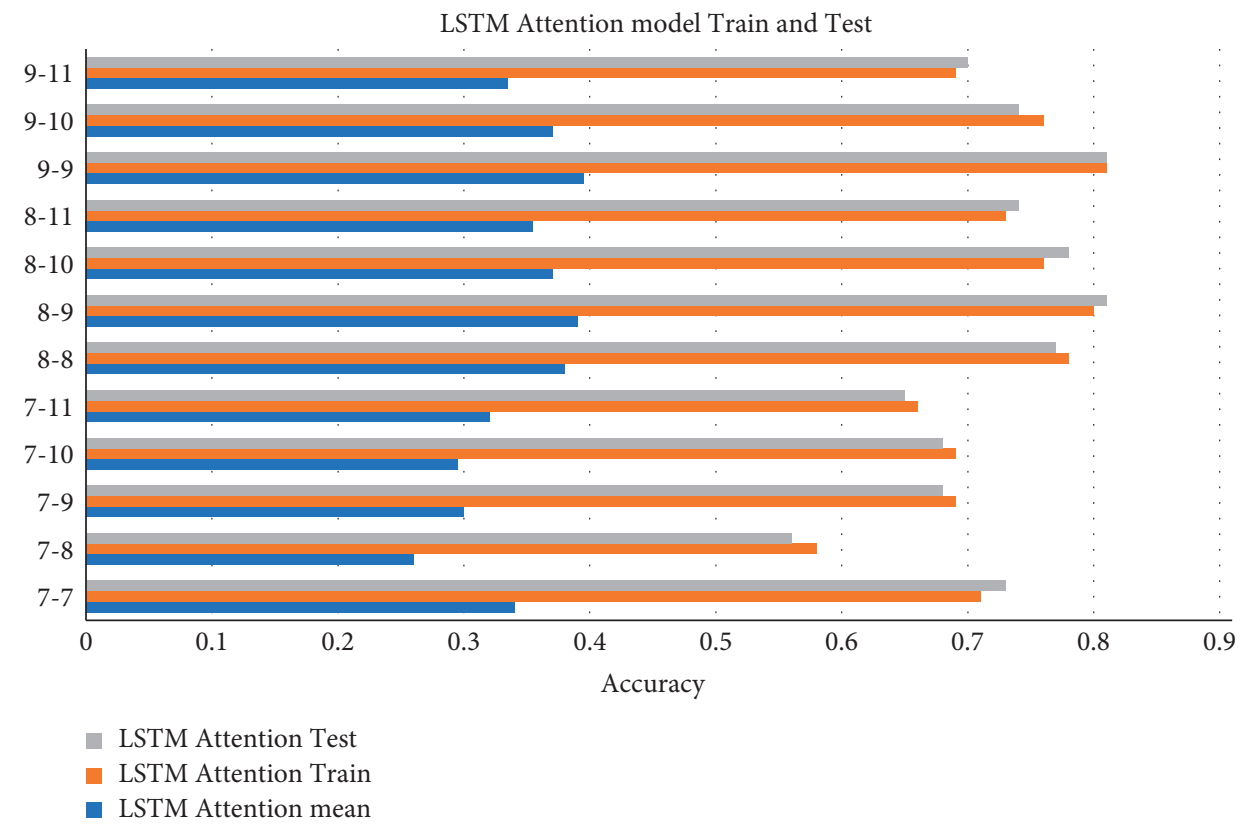

FIgURE 6: Comparison of training and testing of LSTM attention model.

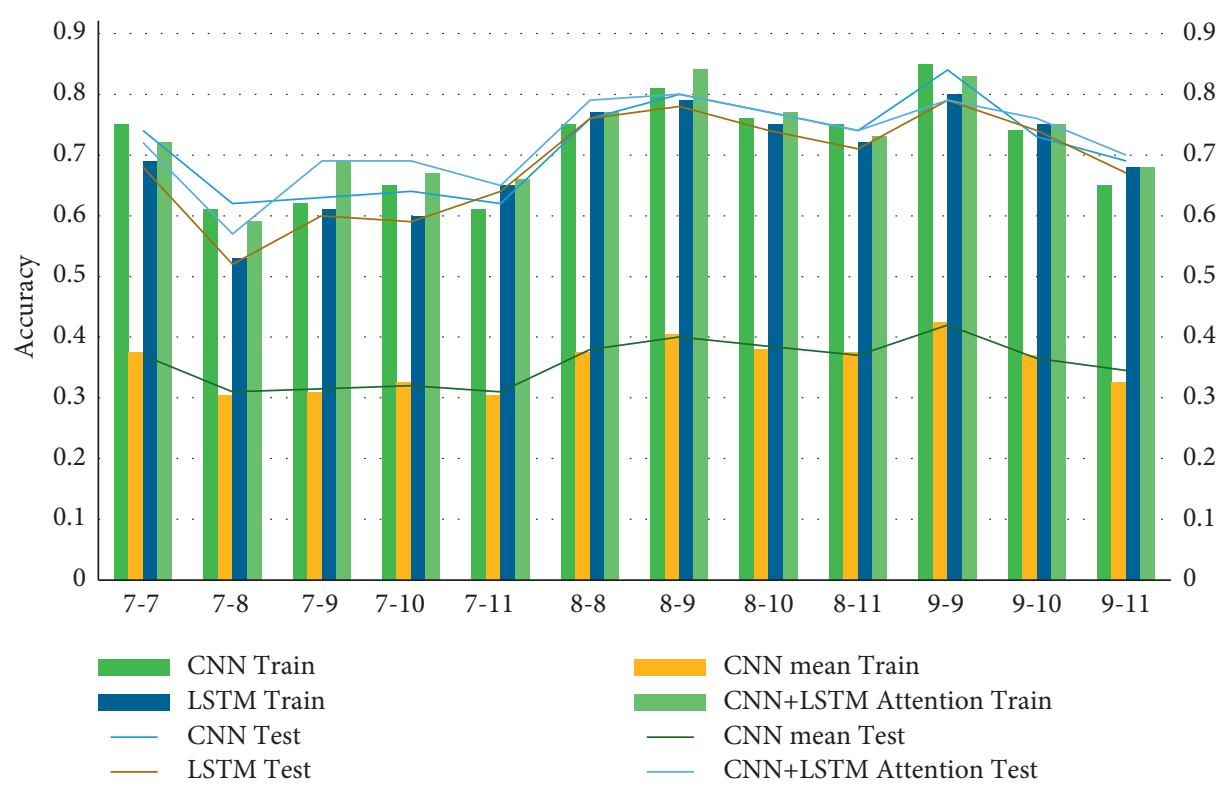

Figure 7: Comparison of training and testing of CNN + LSTM attention model.

4.2. Use Models to Fit Marketing Data. Using the marketing data of W company in previous years, bring in CNN model, LSTM model, LSTM attention model, and CNN + LSTM attention model, and evaluate the model using $\mathrm{Sn}(\%), \mathrm{Sp}(\%)$, Acc(\%), and MCC indicators. As shown in Table 2 and Figure 8, looking at the four indicators of Sn (\%), Sp (\%), Acc (\%), and MCC, the Sn (\%) of the CNN + LSTM attention model $=70.71, \quad$ Sp $\quad(\%)=86.25, \quad \operatorname{Acc}(\%)=81.07, \quad$ and MCC $=0.752$ is the best fitting model.

As shown in Table 3, using CNN + LSTM attention to filter user tags, it is predicted that those users have greater buying potential.
As shown in Table 4, the users are classified by gender and gender, and it is found that men in the $\mathrm{W}$ company are the main purchasing power, and in the age stratification, it is found that the population of 41-50 accounts for the highest proportion. It can be seen from this that when performing precision marketing, the gender and age of the population must be precisely controlled.

4.3. Prediction of Precision Marketing and General Marketing. As shown in Table 5 and Figure 9, in the marketing input forecast for the next year, it is found that the use of precision 
TABLE 2: Fitting of the model to marketing data.

\begin{tabular}{lcccr}
\hline Method & CNN & LSTM & LSTM attention & CNN + LSTM attention \\
\hline Sn $(\%)$ & 69.64 & 63.57 & 46.07 & 70.71 \\
Sp $(\%)$ & 90 & 94.46 & 93.92 & 86.25 \\
Acc(\%) & 83.21 & 84.16 & 77.97 & 81.07 \\
MCC & 0.756 & 0.708 & 0.384 & 0.752 \\
\hline
\end{tabular}

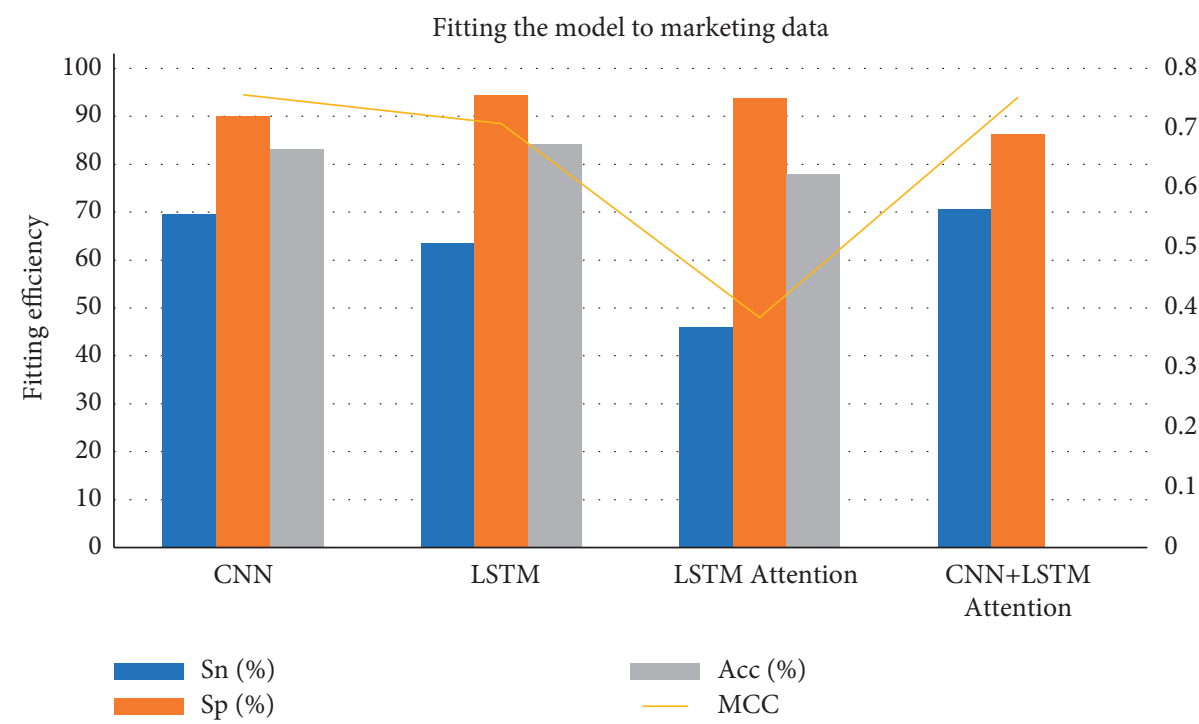

FIGURE 8: Fitting of the model to marketing data.

TABLE 3: Using CNN + LSTM attention to predict the number of users.

\begin{tabular}{lcccc}
\hline Serial number & Original log size $(\mathrm{G})$ & Number of original tags & Number of filtered tags & User number \\
\hline 1 & 19.82 & 2319905 & 487180 & 154727 \\
2 & 40.35 & 4450395 & 934583 & 1444777 \\
3 & 59.64 & 6879890 & 1938783 & 457493 \\
4 & 78.92 & 8812650 & 2486059 & 58726 \\
5 & 101.52 & 11300270 & 3058762 & 747618 \\
6 & 120.08 & 13298965 & 3094094 & 887931 \\
7 & 141.28 & 15470470 & 1031298 \\
\hline
\end{tabular}

TABLE 4: Using CNN + LSTM attention to predict the number of users (male and female stratification and age stratification).

\begin{tabular}{ccccc}
\hline & Attribute value & User number & Percentage of generated data (\%) & Proportion of original data (\%) \\
\hline \multirow{3}{*}{ Gender } & Man & 59190070 & 59.19 & 59.19 \\
& Woman & 32272265 & 32.27 & 32.47 \\
& Others & 8537665 & 8.54 & 8.54 \\
\hline \multirow{4}{*}{ Age } & $0-24$ & 17879563 & 17.88 & 17.88 \\
& $25-30$ & 15928089 & 15.93 & 15.93 \\
& $31-35$ & 10995266 & 11.00 & 11.00 \\
& $36-40$ & 9691802 & 9.69 & 9.69 \\
& $41-50$ & 19059443 & 19.06 & 19.06 \\
& $>50$ & 17924356 & 8.92 & 17.92 \\
\end{tabular}

marketing will increase the profit of $W$ enterprise. The average annual data shows that the monthly income of precision marketing is 0.735 , while that of ordinary marketing is only 0.567 .
4.4. Practical Effects of the Model. After downloading the data of W company from July 1 to November 30, 2020, the original data of $\mathrm{W}$ company's marketing income through different methods is classified, and the comparative 
TABle 5: Prediction of precision marketing and general marketing.

\begin{tabular}{lcc}
\hline Month & Precision marketing & General marketing \\
\hline 1 & 0.73 & 0.5 \\
2 & 0.75 & 0.51 \\
3 & 0.68 & 0.51 \\
4 & 0.65 & 0.49 \\
5 & 0.77 & 0.68 \\
6 & 0.81 & 0.66 \\
7 & 0.78 & 0.62 \\
8 & 0.74 & 0.59 \\
9 & 0.81 & 0.71 \\
10 & 0.74 & 0.58 \\
11 & 0.72 & 0.56 \\
12 & 0.64 & 0.4 \\
\hline
\end{tabular}

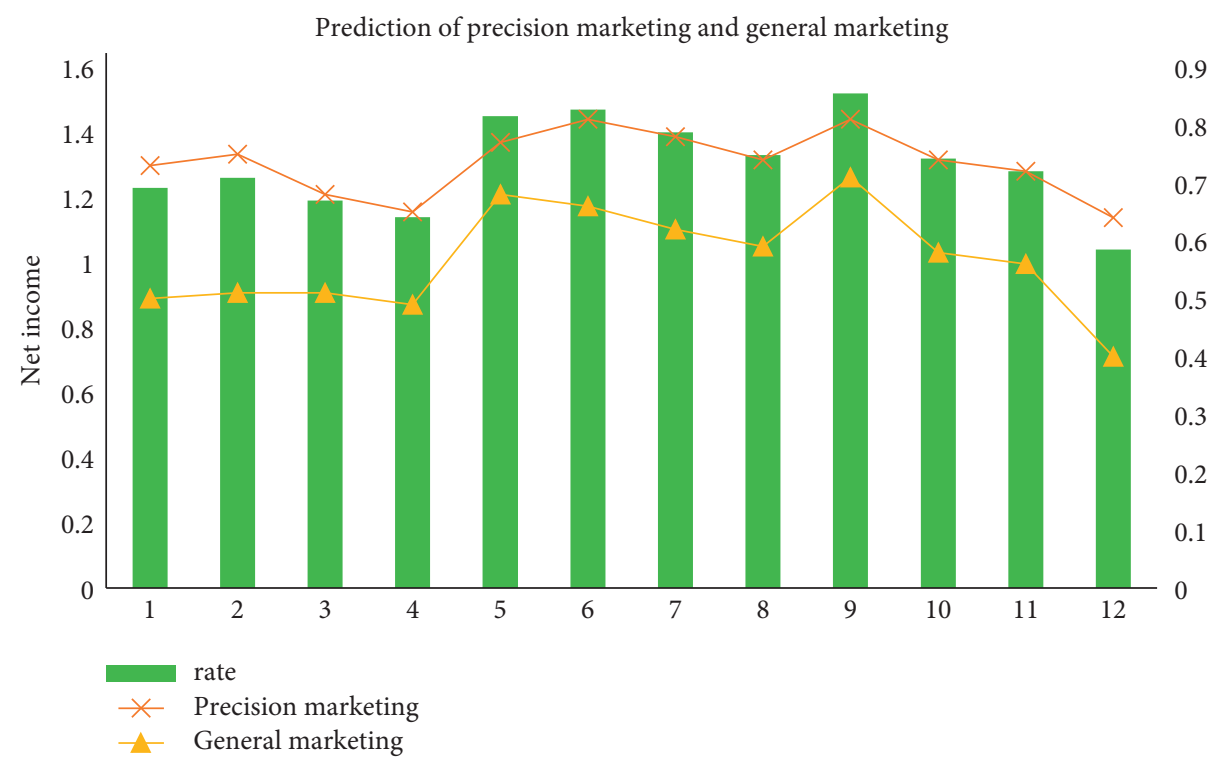

FIGURE 9: Prediction of precision marketing and general marketing.

TABle 6: Practical effects of the model.

\begin{tabular}{lccc}
\hline Months & Precision $\%$ & Recall \% & F1-score \% \\
\hline 7 & 73.1 & 77.6 & 75.2 \\
8 & 61 & 72.3 & 66.2 \\
9 & 55.9 & 91.6 & 69.4 \\
10 & 78.3 & 84.1 & 81.1 \\
11 & 64.9 & 85.7 & 73.8 \\
Mean & 66.6 & 82.3 & 73.1 \\
\hline
\end{tabular}

analysis and supplementary analysis are applied to the optimization model. As shown in Table 6 and Figure 10, the results of comparative analysis and supplementary analysis are displayed. Precision, recall, and F1 scores are used to evaluate the comparative analysis and supplementary analysis, and the comparative analysis and supplementary analysis are simulated. The average accuracy rate of additive analysis is as high as $66.6 \%$, the average recall rate of comparative analysis and complementary analysis is $82.3 \%$, and the F1 score of comparative analysis and complementary analysis is $73.1 \%$. This model has met expectations for precision marketing forecasts. Using precision, recall, and F1 scores to predict the $\mathrm{W}$ company, it is found that the CNN + LSTM attention model can increase monthly revenue by $50 \%$ in peak seasons and about $20 \%$ in off-season. 


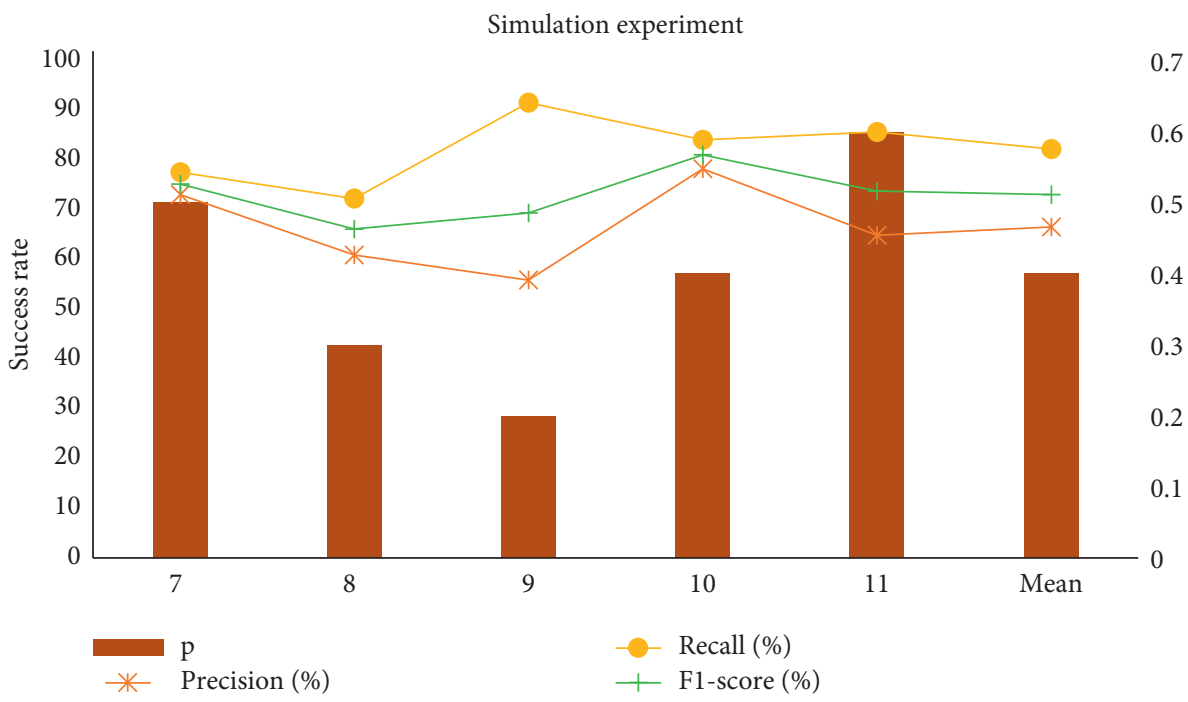

Figure 10: The practical effect of the model.

\section{Conclusion}

This research is based on curling neural networks, precision marketing, model optimization and construction, comparative analysis, and supplementary analysis and proposes precision marketing in response to the problems of existing marketing methods. Optimize the existing curl neural network model and apply it to e-commerce precision marketing. Through the collection and analysis of W enterprise data, the training and testing of CNN model, LSTM model, LSTM attention model, and CNN + LSTM attention model are compared, and the performance of CNN + LSTM attention model and LSTM attention model is obtained. Better, the accuracy of testing and training is higher. By fitting the model, it is found that $\mathrm{Sn}(\%)=$ $70.71, \operatorname{Sp}(\%)=86.25, \operatorname{Acc}(\%)=81.07$, and $\mathrm{MCC}=0.752$ of the CNN + LSTM attention model are the best fitting models. The men and women stratification and gender stratification of users are predicted, and it is found that men in the $\mathrm{W}$ company are the main purchasing power, and in the age stratification, it is found that the population of 41-50 accounts for the highest proportion. Using the CNN + LSTM attention model to predict the marketing input for the next year, it is found that the use of precision marketing will increase the profit of $\mathrm{W}$ company. Using the CNN + LSTM attention model to predict the marketing input for the next year, it is found that the use of precision marketing will increase the profits of $W$ companies. The average annual data show that precision marketing will increase monthly revenue by $42.5 \%$. The simulation shows that the average accuracy rate of comparative analysis and supplementary analysis is as high as $66.6 \%$, the average recall rate of comparative analysis and supplementary analysis is $82.3 \%$, and the $\mathrm{F} 1$ score of comparative analysis and supplementary analysis is $73.1 \%$. The model predicts precision marketing as expected.

\section{Data Availability}

The experimental data used to support the findings of this study are available from the corresponding author upon request.

\section{Conflicts of Interest}

The author declares no conflicts of interest regarding this work.

\section{Acknowledgments}

This work was sponsored in part by the National Social Science Foundation(20BGL176).

\section{References}

[1] X. I. Xian-Ming and L. Wang, "The era of E-commerce marketing development," Journal of Harbin University of Commerce(Social Science Edition), vol. 93, no. 5, pp. 58-67, 2006.

[2] M. Sawhney, "Precision marketing: the new rules for attracting, retaining, and leveraging profitable customers," Akuntansi Pegawai, vol. 93, no. 3, pp. 158-165, 2004.

[3] Z. Y. Liu, "Study on precision marketing method," Journal of Shanghai Jiaotong University, vol. 56, no. 65, pp. 32-45, 2007.

[4] Z. Zhuo, S. Chen, and K. Y. Chau, "A new model of manufacturer's optimal product supply strategy in the context of precision marketing: based on real demand pattern," Mathematical Problems in Engineering, vol. 59, no. 02, pp. 62-98, 2020.

[5] Z. Tian, "Research and analysis of automobile precision marketing based on big data:taking the construction of Audi car user portrait as an example," Journal of Fujian University of Technology, vol. 17, no. 4, pp. 391-397, 2019.

[6] T. Cai and Z. Zhao, "Convolutional neural network-based surgical instrument detection," Technology and Health Care, vol. 28 , no. S1, pp. 81-88, 2020.

[7] S. Harada, H. Akita, M. Tsubaki et al., "Dual graph convolutional neural network for predicting chemical networks," BMC Bioinformatics, vol. 21, no. Suppl 3, p. 94,, 2020 Apr 23.

[8] K. Zhao and C. Wang, "Sales forecast in E-commerce using convolutional," Neural Network[J], vol. 156, no. 51, pp. 21-46, 2017.

[9] J. Hochuli, A. Helbling, T. Skaist, M. Ragoza, and D. R. Koes, "Visualizing convolutional neural network protein-ligand 
scoring," Journal of Molecular Graphics and Modelling, vol. 84, pp. 96-108,, 2018 Sep.

[10] S. M. Anwar, M. Majid, A. Qayyum, M. Awais, M. Alnowami, and M. K. Khan, "Medical image analysis using convolutional neural networks: a review," Journal of Medical Systems, vol. 42, no. 11, p. 226, 2018 Oct 8.

[11] Z. Cao, S. Mu, and M. Dong, "Two-attribute e-commerce image classification based on a convolutional neural network," The Visual Computer, vol. 94, no. 55, pp. 158-172, 2020.

[12] H. Pan and H. Zhou, "Study on convolutional neural network and its application in data mining and sales forecasting for E-commerce," Electronic Commerce Research, vol. 12, no. 154, pp. 123-142, 2020.

[13] A. Jx and B. Lw, "Collaborative innovation of E-Commerce enterprises based on FPGA and convolutional neural network - ScienceDirect," Microprocessors and Microsystems, vol. 12, no. 154 , pp. $13-35,2020$.

[14] A. Sun, X. Zhao, and C. Hu, "A novel convolutional neural network approach to improve the multi-product image based classification IN e-commerce," Journal of Shanxi Normal University (Philosophy and Social Sciences edition), vol. 12, no. 4, pp. 36-59, 2018.

[15] A. Schindler, T. Lidy, and S. Karner, "Fashion and apparel classification using convolutional neural networks," Computer Vision and Pattern Recognition, vol. 5, no. 5, pp. 3-34, 2018.

[16] A. Krizhevsky, "One weird trick for parallelizing convolutional neural networks," Eprint Arxiv, vol. 12, no. 14, pp. 62-98, 2014.

[17] G. Suman, A. Panda, P. Korfiatis, and A. H. Goenka, "Convolutional neural network for the detection of pancreatic cancer on CT scans," The Lancet Digital Health, vol. 2, no. 9, p. e453, 2020 Sep.

[18] W. Yin, H. Schütze, and B. Xiang, "ABCNN: attention-based convolutional neural network for modeling sentence pairs," Computer Science, vol. 12, no. 5, pp. 98-122, 2015.

[19] V. Lebedev, Y. Ganin, and M. Rakhuba, "Speeding-up convolutional neural networks using fine-tuned CP-decomposition," Computer Science, vol. 11, no. 14, pp. 123-142, 2014.

[20] A. Dosovitskiy, P. Fischer, and J. T. Springenberg, "Discriminative unsupervised feature learning with exemplar convolutional neural networks," IEEE Transactions on Pattern Analysis and Machine Intelligence, vol. 38, no. 9, pp. 17341747, 2014.

[21] C. Santos, X. Bing, and B. Zhou, "Classifying relations by ranking with convolutional neural networks," Computer Science, vol. 86, no. 86, pp. 132-137, 2015.

[22] P. Molchanov, S. Tyree, T. Karras, T. Aila, and J. Kautz, "Pruning convolutional neural networks for resource efficient transfer learning," Machine Learning, vol. 5, no. 4, pp. 048067, 2017.

[23] G. Chen and S. Li, "Research on location fusion of spatial geological disaster based on fuzzy SVM," Computer Communications, vol. 153, pp. 538-544, 2020.

[24] G. Sun, C.-C. Chen, and S. Bin, "Study of cascading failure in multisubnet composite complex networks," Symmetry, vol. 13, no. 3, p. 523, 2021.

[25] K. Kang, H. Li, and J. Yan, "T-CNN: tubelets with convolutional neural networks for object detection from videos," IEEE Transactions on Circuits and Systems for Video Technology, vol. 28, no. 10, pp. 2896-2907, 2018.

[26] S. Li and A. B. Chan, "3D human pose estimation from monocular images with deep convolutional neural network,"
Asian Conference on Computer Vision, vol. 12, no. 15, pp. 56-72, 2014.

[27] P. Lakhani and B. Sundaram, "Deep learning at chest radiography: automated classification of pulmonary tuberculosis by using convolutional neural networks," Radiology, vol. 3, no. 5, pp. 23-51, 2017. 